\title{
Pituitary metastasis of choriocarcinoma: A case report
}

\author{
HUAN LIU*, YU-DONG XIAO*, SHU-PING PENG, SHUN-KE ZHOU and JUN LIU \\ Department of Radiology, The Second Xiangya Hospital of Central South University, Changsha, Hunan 410011, P.R. China
}

Received September 22, 2014; Accepted June 3, 2015

DOI: $10.3892 / 01.2016 .4088$

\begin{abstract}
Choriocarcinoma is an aggressive obstetric or gynecological neoplasm with a high malignant potential. It is a form of gestational trophoblastic disease and often secondary to hydatidiform mole, and intrauterine or ectopic pregnancy. Choriocarcinoma has a proclivity to metastasize to the lung, vagina, pelvis or liver in over $50 \%$ of patients, which always occurs in the early stage of the disease. With an appropriate amount of chemotherapy, the tumor may be treated effectively. However, pituitary metastasis of choriocarcinoma is extremely rare. To the best of our knowledge, no cases of choriocarcinoma metastasizing to the pituitary have been cited previously. Here, we report a case of choriocarcinoma that presented with pituitary metastasis.
\end{abstract}

\section{Introduction}

Gestational trophoblastic diseases (GTD) are a spectrum of tumors with a variety of biological behaviors and the potential to metastasize. GTDs consist of hydatidiform mole, invasive mole, choriocarcinoma and placental site trophoblastic tumor. Choriocarcinoma is one of the most malignant and aggressive neoplasms of all the GTDs and often secondary to hydatidiform mole (1), and intrauterine or ectopic pregnancy (2-4). Choriocarcinoma is often associated with a high level of $\beta$ human chorionic gonadotrophin ( $\beta-\mathrm{HCG})$ and rapid hematogenous spread to other organs, including the lung, vagina, pelvis and liver (5). However, pituitary metastasis of choriocarcinoma is extremely rare. In this paper we report a case of choriocarcinoma metastases to the pituitary.

Correspondence to: Professor Jun Liu, Department of Radiology, The Second Xiangya Hospital of Central South University, 139 Middle Renmin Road, Changsha, Hunan 410011, P.R. China E-mail: 060145548@qq.com

${ }^{*}$ Contributed equally

Abbreviations: GTD, gestational trophoblastic diseases; $\beta$-HCG, $\beta$ human chorionic gonadotrophin; MR, magnetic resonance

Key words: choriocarcinoma, metastasis, pituitary

\section{Case report}

A 22-year-old female was admitted to The Second Xiangya Hospital of Central South University (Changsha, China), complaining of a headache for 20 days and blurred vision for 10 days. The patient had no vaginal bleeding, cough or hemoptysis. Upon physical examination, she appeared to be in a good general condition with no fever, no hypertension, no signs of meningism and no neurological deficits. Two-and-a-half years previously, the patient had attended the same hospital with a 6-month history of menelipsis and suffering from irregular vaginal bleeding for 20 days. Following relevant examinations, a diagnosis of choriocarcinoma had been made. After administration of a sufficient amount of chemotherapy (15 cycles of $26.36 \mathrm{mg} / \mathrm{kg}$ tegafur, $6.43 \mu \mathrm{g} / \mathrm{kg}$ actinomycin D and $20 \mathrm{mg}$ methylaminopterin per day, with each cycle lasting 7 days), the patient's condition improved. During the 2 years of follow-up, the patient had remained in a good condition and her blood $\beta-\mathrm{HCG}$ remained at the normal level.

On the present occasion, the patient complained of a swelling pain in the head, which had commenced 20 days previously. The pain lasted for a few seconds, and was particularly notable in the early morning. In addition, the patient was suffering from blurred vision which had persisted for 10 days. No mass was identified in the pelvis or lung upon whole-body computed tomography examination. A head magnetic resonance (MR) examination was performed, which revealed a lobulated mass with an irregular shape located in the suprasellar cistern. The maximum diameter of the mass was $\sim 4 \mathrm{~cm}$ and the hypophyseal stalk demonstrated notable thickening, indicating pituitary metastasis of choriocarcinoma. The mass had iso-signal intensity on T1-weighted imaging (T1WI) and slightly high signal intensity on T2WI. A T2WI fluid-attenuated inversion recovery sequence revealed that the mass had irregular slightly high signal intensity with mild edema in the peritumoral region. Following contrast agent injection, heterogeneous enhancement was noted in the mass (Fig. 1). In the blood laboratory examination, the $\beta$-HCG level was extremely high, at a level of $3899.000 \mathrm{mIU} / \mathrm{ml}$ (the normal serum $\beta-\mathrm{HCG}$ level is $<100 \mathrm{mIU} / \mathrm{ml}$ in non-pregnant females). A diagnosis of pituitary metastasis of choriocarcinoma was made. Following the administration of 6 seven-day cycles of chemotherapy (6 cycles of $100 \mathrm{mg} / \mathrm{m}^{2}$ etoposide, $6.43 \mu \mathrm{g} / \mathrm{kg}$ actinomycin D and $80 \mathrm{mg} / \mathrm{m}^{2}$ cisplatin per day, with each cycle lasting 7 days), the patient's symptoms were relieved and her $\beta-\mathrm{HCG}$ returned to a normal level. The patient underwent a second 


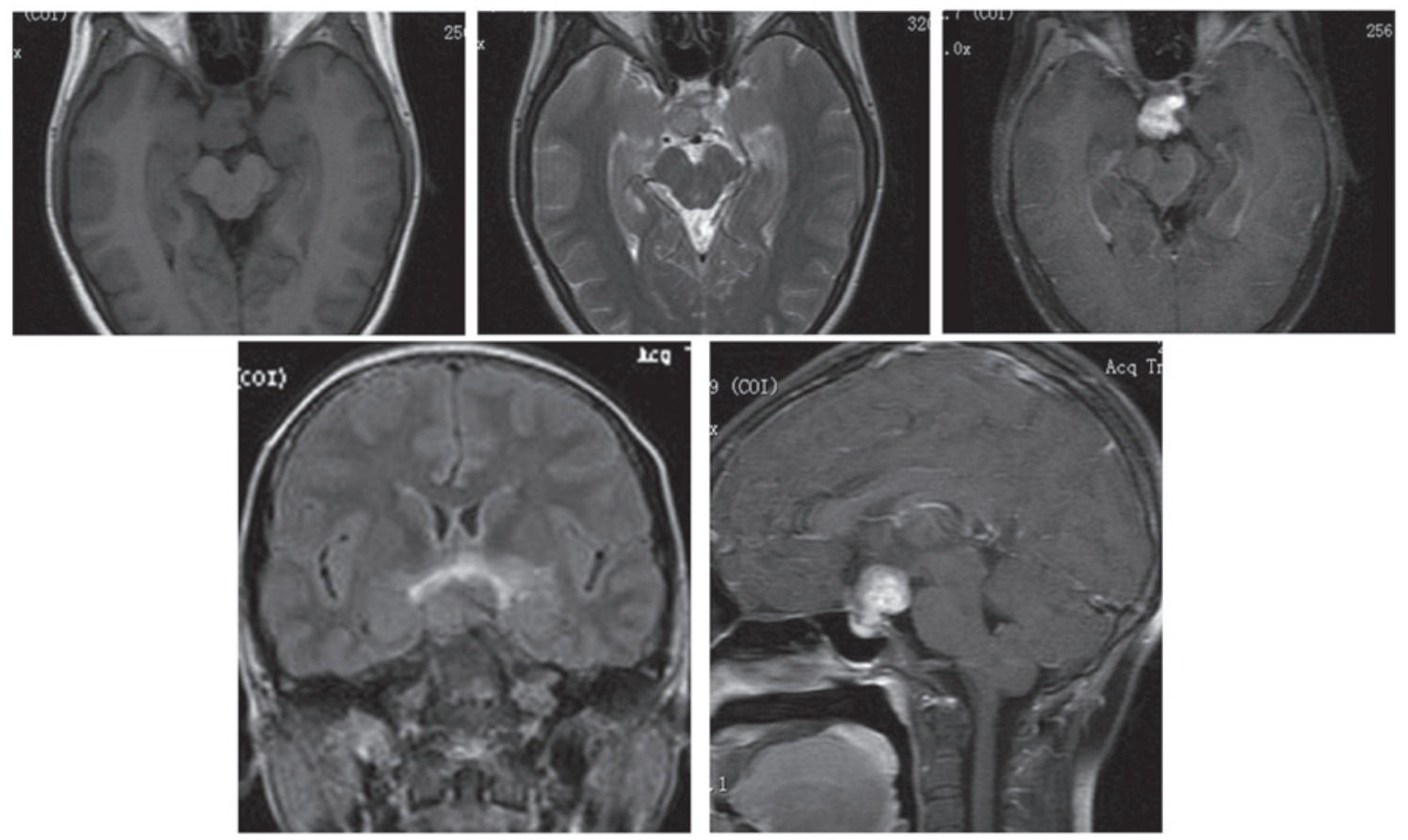

Figure 1. Head magnetic resonance examination revealed that an irregular shape located in the suprasellar cistern. The mass had an iso-signal on T1-weighted imaging and a slightly high signal on T2-weighted imaging. T2 fluid-attenuated inversion recovery revealed that the mass had mild edema. Following injection of contrast agent, heterogeneous enhancement was noted.
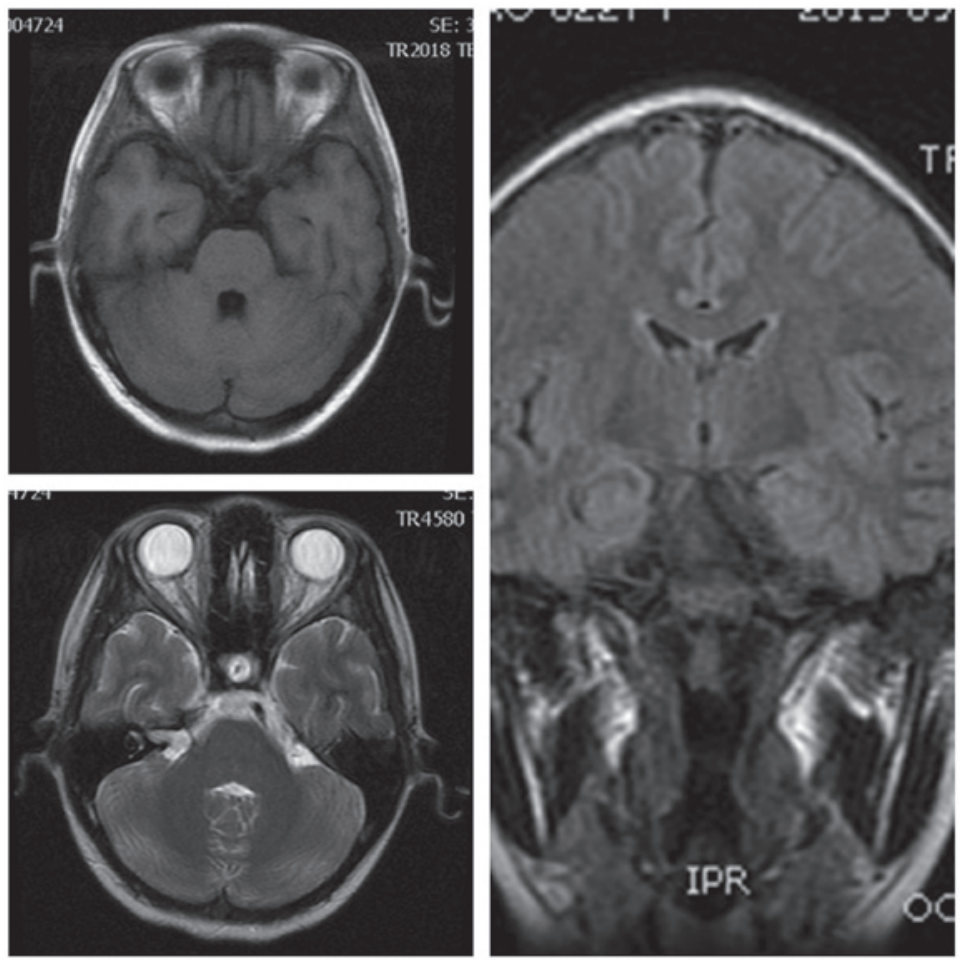

Figure 2. After two months, the patient underwent a second head magnetic resonance examination, which revealed that the mass was no longer present and that the sellar and suprasellar structures were clear.

head MR examination after 2 months, which revealed that the mass was no longer present and that the sellar and suprasellar structures were clear (Fig. 2). Eight months later, a third head MR examination was performed, which revealed no tumor recurrence in the suprasellar cistern (Fig. 3). Subsequently, follow-up MR examinations every 6 months were planned.
At the most recent follow-up, conducted on June 17, 2013, the patient was well and no recurrent lesions were identified.

The patient provided written informed consent for the current case report, and ethical approval was obtained from the ethics committee of the Second Xiangya Hospital of Central South University. 

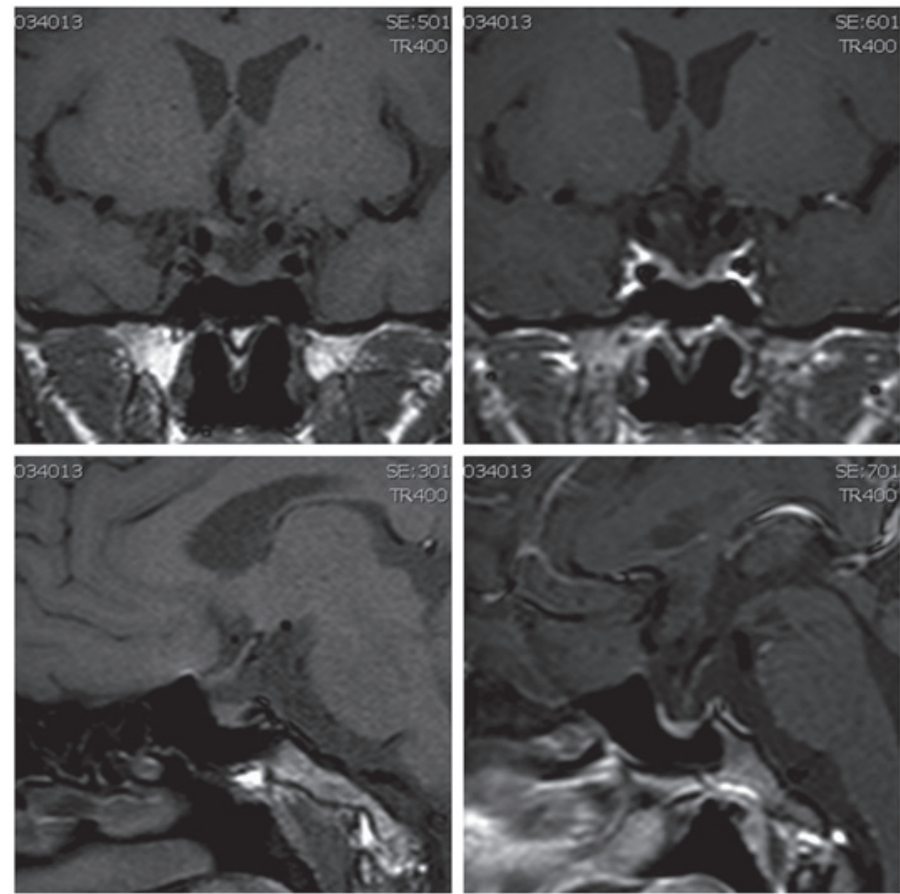

Figure 3. Eight months later, a third head magnetic resonance examination was performed which demonstrated no tumor recurrence in the suprasellar cistern.

\section{Discussion}

GTDs consist of a group of neoplastic disorders that arise from the trophoblastic epithelium of the placenta (6). On the basis of histopathological classification, GTDs may be divided into five types: complete and partial hydatidiform mole, invasive mole, choriocarcinoma and placental site trophoblastic tumor. They have distinct tumor markers, including $\beta$-HCG (7). Choriocarcinoma is one of the most common types of GTD, and has a high malignant potential (8). This tumor is associated with molar pregnancy and rapid hematogenous spread to other organs. It is widely believed that pregnancy is a high risk factor for the development of choriocarcinoma. It has been reported that $\sim 50 \%$ of choriocarcinoma cases arise in molar pregnancy, $25 \%$ arise following previous abortions, $22.5 \%$ arise in normal pregnancy and $2.5 \%$ arise subsequent to ectopic pregnancy (9).

Choriocarcinoma is frequently associated with multiple-organ metastasis, which often occurs in the early stage of the disease with a generally hematogenous spreading pattern due to the affinity of trophoblasts for blood vessels (10). The organs commonly affected by choriocarcinoma metastasis include the lung, vagina, pelvis and liver (5). Other organs including the brain, lumbar, bone and sacrum have occasionally been reported to develop metastases (11). To the best of our knowledge, no cases of choriocarcinoma metastasizing to the pituitary have been cited previously. In this case, we did not have pathological evidence to confirm the diagnosis of choriocarcinoma. However, pathological evidence of choriocarcinoma is not a necessary requirement for making a final diagnosis (12). The blood $\beta$-HCG level and the clinical symptoms of patients are more significant in this respect. The first time that our patient visited the hospital, her blood $\beta$-HCG level was at a high level. Following regular chemotherapy, her $\beta$-HCG descended to a normal level. However, after 2 years of follow-up, her blood $\beta$-HCG returned to a high level once again, indicating a recurrence of the tumor. A head MR examination was performed which revealed a pituitary mass associated with the choriocarcinoma. After administration of regular chemotherapy, the mass disappeared. For this reason, a diagnosis of pituitary metastasis of choriocarcinoma could be made.

Although choriocarcinoma has a high malignant biological behavior, it demonstrates a good response to chemotherapy (13). It was demonstrated that the chemotherapy effectively keeps the pituitary metastasis of choriocarcinoma under control, thereby avoiding the overall need for radiation (14). One study has reported that chemotherapy can treat choriocarcinoma successfully even in the advanced stage (15). In the present case, the pituitary metastasis of choriocarcinoma disappeared following a quantity of regular chemotherapy.

In conclusion, pituitary metastasis of choriocarcinoma is extremely rare. With an appropriate amount of chemotherapy, tumors may be successfully treated. In addition, a final diagnosis should be considered comprehensively based not only on imaging findings, but also on laboratory examinations and clinical symptoms.

\section{References}

1. Dhrami-Gavazi E, Lo C, Patel P, Galic V, Pareja F and Kazim M: Gestational choriocarcinoma metastasis to the extraocular muscle: A case report. Ophthal Plast Reconstr Surg 30: e75-e77, 2014.

2. Seck1 MJ, Sebire NJ and Berkowitz RS: Gestational trophoblastic disease. Lancet 376: 717-729, 2010

3. Zhang F, Zhang W, Shi H, Ye G, Shi W, Shu Y and Li G: Primary choriocarcinoma of the posterior mediastinum in a male: A case report and review of the literature. Oncol Lett 8: 739-741, 2014.

4. Liu Y, Yang J, Ren T, Zhao J, Feng F, Wan X and Xiang Y: The encouraging prognosis of nongestational ovarian choriocarcinoma with lung metastases. J Reprod Med 59: 221-226, 2014. 
5. Sekine R, Hyodo M, Kojima M, Meguro Y, Suzuki A, Yokoyama T, Lefor AT and Hirota N: Primary hepatic choriocarcinoma in a 49-year-old man: report of a case. World J Gastroenterol 19 : 9485-9489, 2013.

6. Kobayashi Y, Masuda K, Banno K, Kobayashi N, Umene K, Nogami Y, Tsuji K, Ueki A, Nomura H, Sato K, et al: Glycan profiling of gestational choriocarcinoma using a lectin microarray. Oncol Rep 31: 1121-1126, 2014.

7. Guo J, Zhong C, Liu Q, Xu J, Zheng Y, Xu S, Gao Y, Guo Y, Wang Y, Luo Q, et al: Intracranial choriocarcinoma occurrence in males: Two cases and a review of the literature. Oncol Lett 6: 1329-1332, 2013.

8. Hsieh YT, Chou MM, Chen HC and Tseng JJ: IMP1 promotes choriocarcinoma cell migration and invasion through the nove effectors RSK2 and PPME1. Gynecol Oncol 131: 182-190, 2013.

9. Braun-Parvez L, Charlin E, Caillard S, Ducloux D, Wolf P, Rolle F, Golfier F, Flicoteaux H, Bergerat JP and Moulin B: Gestational choriocarcinoma transmission following multiorgan donation. Am J Transplant 10: 2541-2546, 2010.

10. Müller CSL, Tilgen W, Pföhler C and Graf N: Cutaneous and systemic metastasis of a testicular choriocarcinoma. Am J Dermatopathol 32: 521-522, 2010.
11. Cong Q, Li G, Jiang W, Li B, Wang Y, Yao L, Wang S and $\mathrm{Xu}$ C: Ectopic choriocarcinoma masquerading as a persisting pregnancy of unknown location: Case report and review of the literature. J Clin Oncol 29: e845-e848, 2011.

12. Liu X, Li X, Yin L, Ding J, Jin H and Feng Y: Genistein inhibits placental choriocarcinoma cell line JAR invasion through ER $\beta /$ MTA3/Snail/E-cadherin pathway. Oncol Lett 2: 891-897, 2011.

13. Dadlani R, Furtado SV, Ghosal N, Prasanna KV and Hegde AS: Unusual clinical and radiological presentation of metastatic choriocarcinoma to the brain and long-term remission following emergency craniotomy and adjuvant EMA-CO chemotherapy. J Cancer Res Ther 6: 552-556, 2010.

14. Ryu JH, Choi CH, Kim TJ, Lee JW, Kim BG and Bae DS: Chemo-resistant choriocarcinoma metastatic to colon cured by low-anterior resection. J Gynecol Oncol 22: 203-206, 2011.

15. Kim M, Yun J, Hur SM, Moon JJ, Nam JH, Kim SH, Kim HJ, Kim CK, Park SK and Hong DS: Successful synchronous chemotherapy and radiotherapy followed by consecutive chemotherapy without surgery for primary intracranial choriocarcinoma: A case report. Oncol Lett 4: 1389-1391, 2012. 